\title{
EFFECT OF HOM COUPLERS ON THE ACCELERATING MODE IN THE DAMPED CAVITY AT THE PHOTON FACTORY STORAGE RING
}

\author{
T. Takahashi", M. Izawa, S. Sakanaka, and K. Umemori, High Energy Accelerator Research \\ Organization (KEK), 1-1 Oho, Tsukuba, Ibaraki 305-0801, Japan \\ T. Koseki, RIKEN, Saitama 351-0198, Japan.
}

\begin{abstract}
Effect of a rod type HOM coupler on the accelerating mode in a normal conducting single cell cavity was studied by using MAFIA. Usually, this type of coupler is used to reduce Q-values of some HOMs such as TM011, TM111, TE111, TM021-like modes which have strong Efield in the middle of cylindrical wall of the cavity. We are trying to use this rod type HOM coupler in the offcentred position on the cylindrical wall in order to make it effective for other HOMs such as TM012, TM022-like modes. When it is attached to the position apart from the middle of the cylindrical wall, the coupling with the fundamental mode should be taken into account. Therefore we studied the effect of the rod type HOM coupler on the fundamental mode by the use of MAFIA. The results are presented.
\end{abstract}

\section{INTRODUCTION}

The rod type HOM coupler is one of well known rf technology [1]. It is effective to reduce Q-values of popular HOMs such as TM011, TM111, TE111, TM021like modes. As compared with the loop type HOM coupler, it is easy to use since it does not couple with accelerating mode when it is attached to the cavity properly in the middle of the cylindrical wall. As for other annoying HOMs such as TM110, TM020-like modes, the rod type coupler has no coupling with them. However, TM020, TM012-like modes can be well damped by the rod type coupler when it is placed in the off-centred position on the cylindrical wall. On the other hand, in such off-centred position, the electromagnetic field of fundamental mode around rod antenna becomes asymmetric resulting non negligible coupling between the HOM coupler and the accelerating mode. Therefore we investigated the effect of the rod type HOM coupler on the accelerating mode by using MAFIA. In this paper, we concentrate in the rod type HOM coupler and call it simply a damper. We call it an off-centred damper when it is attached to the location apart from the middle of the cylindrical wall of the cavity and a normal damper when it is attached to the middle of cylindrical wall. The cavity discussed here is now under manufacturing and will be installed in the Australian Synchrotron Project ring [2].

\section{EFFECT OF DAMPER}

\section{Normal damper}

Figure 1 shows a cross sectional view of a damper. It has a rod antenna followed by coaxial waveguide. HOM

\footnotetext{
\#takeshi.takahashi@kek.jp
}

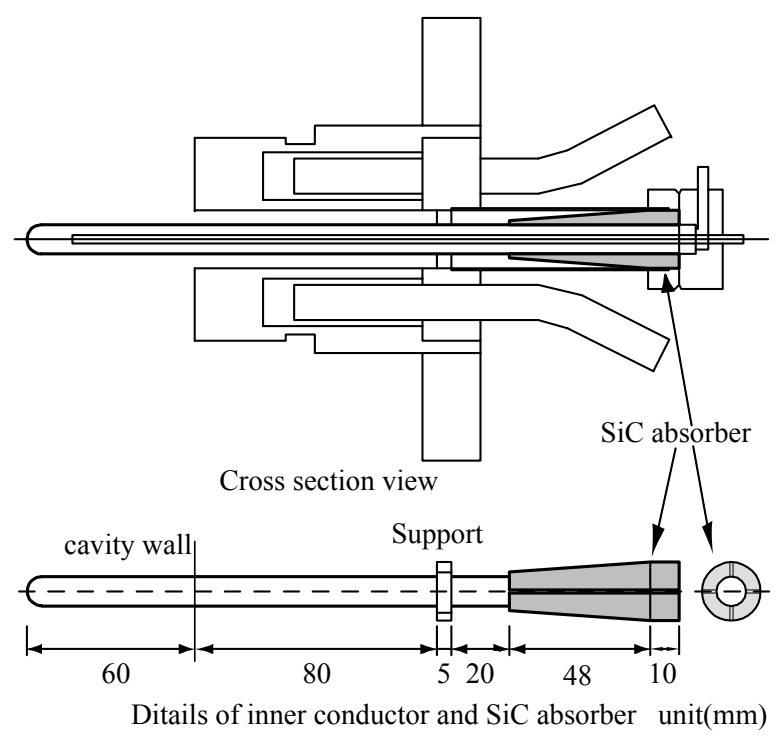

Figure 1: Cross section view of the HOM damper

absorber made of $\mathrm{SiC}$ (silicon carbide) is placed at the end of coaxial waveguide. The shape of $\mathrm{SiC}$ is optimized to reduce VSWR [3]. The effect of a damper on the fundamental mode is simple if it is attached to the middle of cylindrical wall of the cavity. The coaxial mode of the
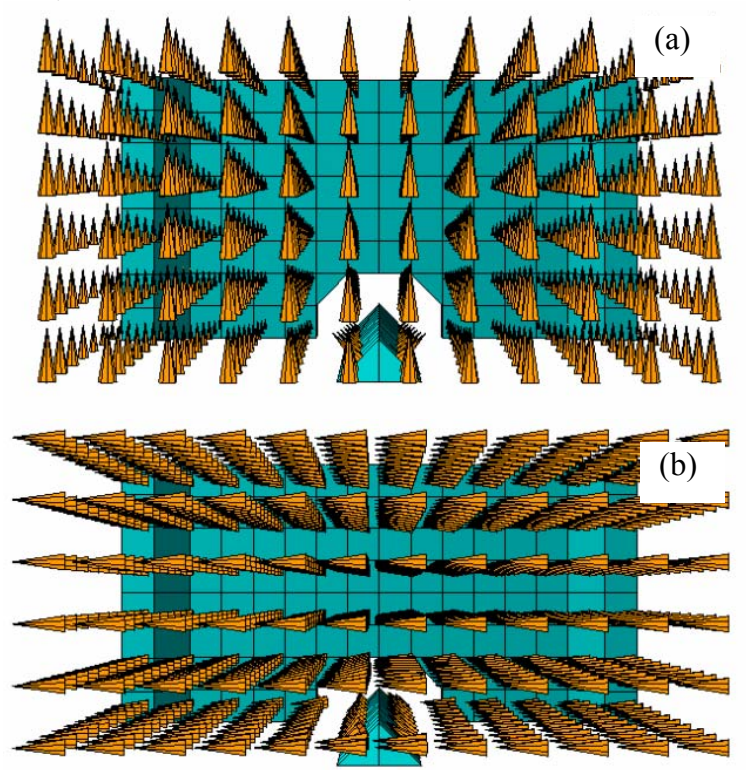

Figure 2: The electromagnetic field of the fundamental mode around the rod antenna. (a) electric field. (b) magnetic field. 


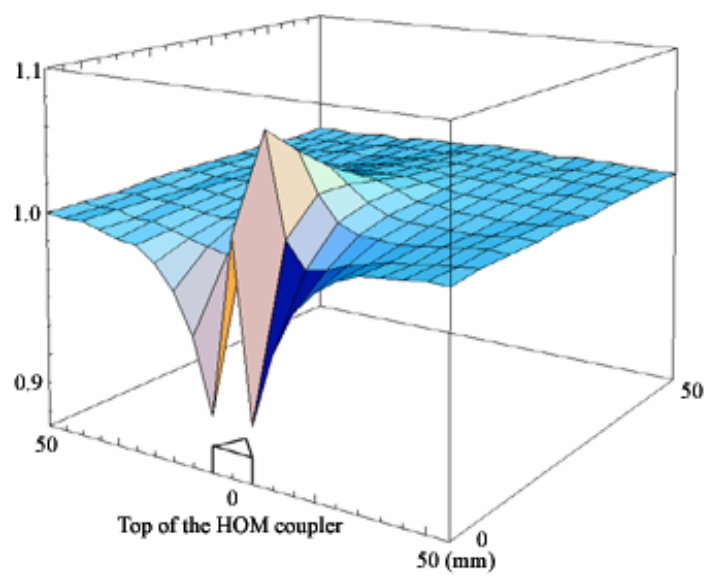

Figure 3: The magnitude of electric field around the top of the rod antenna. The electric field is disordered by about $7 \%$.

damper does not couple with the fundamental mode. Its effect is local as expected. The electromagnetic field of the fundamental mode is slightly disordered around the rod antenna as shown in Fig. 2 and 3. From the practical point of view, it seems to be no serious problem for this type of damper. Concentration of the electric field, however, on the surface of the rod antenna should be taken into account. Calculation was carried out up to the length of the rod antenna of $80 \mathrm{~mm}$. The strength of the electric field at the top of antenna becomes more than $45 \%$ of that of the nosecone in this case. It is recommended that the length of antenna is below $60 \mathrm{~mm}$ in our cavity. The results of calculation for two dampers which are placed at 90 degree apart in the cavity shows that small amount of multipole coefficients arises in the beam axis of cavity. However, it is very weak and probably the effect should be considered together with that of the input coupler which is out of calculation in this study.

\section{Off-centred damper}

The calculation for the off-center damper was carried

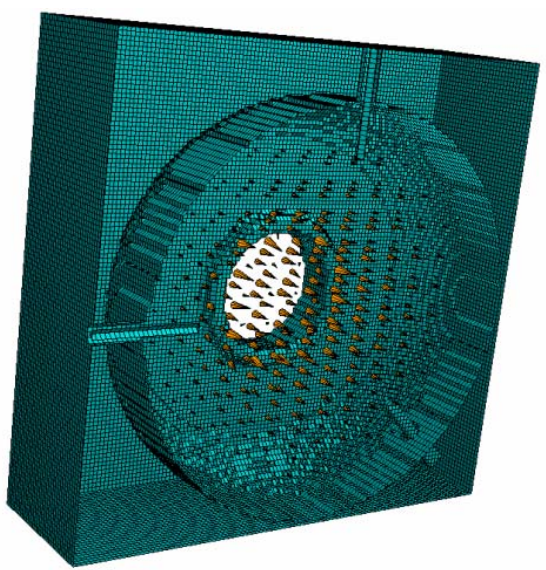

Figure 4: The calculated cavity shape with three HOM couplers. The length of the rod antenna is 60 $\mathrm{mm}$.

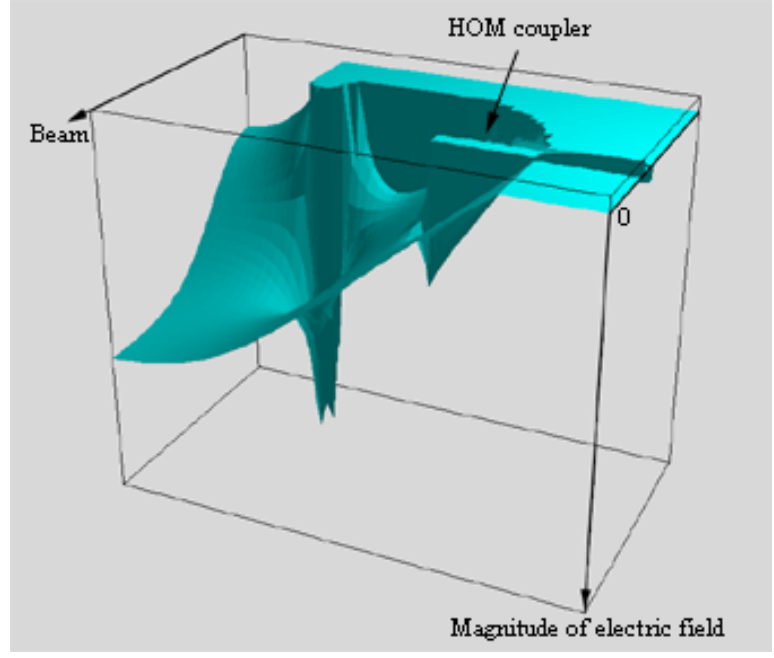

Figure 5: The distribution of the magnitude of electric field around the off-centred damper.

out for two positions of 40 and $60 \mathrm{~mm}$ apart from the middle of cylindrical wall. Figure 4 shows the cavity with three dampers. Three rod antennas have the same length of $60 \mathrm{~mm}$. The distinguishing characteristics among these couplers are that the TEM mode is excited in the coaxial part of the off-centred damper as shown in Fig. 5. The strength of the mode becomes larger at the position of 60 $\mathrm{mm}$ out of the middle. The electromagnetic field of the fundamental mode is also disordered around the rod antenna. However, the strength of the field is the similar level with the normal damper. Therefore, the extent of penetration of the fundamental mode into the off-centred damper is essential. It is necessary to make a careful measurement of the coupling between the off-centred damper and the fundamental mode. The distance from the middle of cavity is an important point.

\section{SUMMARY}

The effect of the rod type damper on the accelerating mode in a normal conducting single cell cavity was studied by using MAFIA. There seems no serious problem. However, when it is attached to the cavity at offcentred position, the coupling between the fundamental mode and the coaxial mode of the coupler becomes non negligible. Careful measurement should be necessary.

\section{REFERENCES}

[1] T. Koseki et al., "Coaxial HOM coupler for the $500 \mathrm{MHz}$ RF damped cavity", Nucl. Instr. Methods, A 467-468, 2001, p. 91.

[2] J. Watanabe et al., "Design and Cold Model Test of $500 \mathrm{MHz}$ Damped Cavity for ASP Storage Ring rf System", in this conference.

[3] T. Takahashi et al., "Design of a HOM Coupler for a Damped Cavity at the Photon Factory Storage Ring", PAC99, New York, 1999, p. 904. 